\title{
Online Measurement of LHC Beam Parameters with the ATLAS High-Level Trigger
}

\author{
D. W. Miller, on behalf of the ATLAS collaboration
}

\begin{abstract}
We present the results of the first online measurement in ATLAS of the LHC beam position and size at $\sqrt{s}=900 \mathrm{GeV}$ in 2009 and $\sqrt{s}=7 \mathrm{TeV}$ in spring 2010. A dedicated algorithm, implemented in the ATLAS Level 2 Trigger, takes fully reconstructed tracks in the Inner Detector as input to a fast vertex fitter in order to reconstruct vertices on an eventby-event basis. The three-dimensional distribution of primary vertices carries information of the $\mathrm{LHC}$ luminous region at the ATLAS interaction point and is used to extract its position, size and tilt angles. The luminous region parameters are monitored in real-time and used for feedback to the LHC. With this method, we observe changes in the transverse centroid position that mirror IP-orbit drifts, as well as longitudinal shifts arising from RF phase changes. Also, variations in the transverse widths, and an expected increase in the longitudinal spot size over the course of a fill were seen. In addition, the measured beam spot is used to track significant changes in the accelerator, which can then be redistributed to the High-Level Trigger for use by trigger algorithms that depend on the precise knowledge of impact parameter or decay length, such as b-tagging. We will present the techniques developed to allow such real-time configuration changes on the High-Level Trigger farm of currently 810 processing nodes in a way that does not disrupt data taking or incur deadtime, while ensuring a consistent and reproducible configuration across the farm. Lastly, by counting the primary vertices online, we use this same algorithm to provide online monitoring of the instantaneous luminosity of the accelerator. The beam position measurements presented here were available in real-time and used to provide feedback to the LHC operators for beam adjustments during the first $L H C$ runs.
\end{abstract}

\section{INTRODUCTION}

A TLAS [1] is one of the two general purpose detectors in operation at the Large Hadron Collider (LHC) at CERN [2]. Built for both precision Standard Model measurements as well as for searches for new phenomena beyond the Standard Model, several technological hurdles face the ATLAS detector. With a nominal $40 \mathrm{MHz}$ proton-proton bunch crossing (BC) rate and approximately 25 proton-proton collisions per $\mathrm{BC}$ at the design luminosity of $10^{34} \mathrm{~cm}^{-2} \mathrm{~s}^{-1}$, the data rate must be reduced by over six orders of magnitude before being written to disk, thereby requiring parallel processing on large compute farms. In addition, many criteria used to decide which events will be recorded are sensitive to changes in the transverse and longitudinal position of the LHC beams and must be updated for any such changes during data taking in order to maintain optimal performance. Such real-time configuration changes require massively parallel operations across many computing nodes with stringent requirements on

D. W. Miller is with the SLAC National Accelerator Laboratory, Menlo Park, CA 94025 USA (e-mail: David.Miller@slac.stanford.edu). This work has been supported in part by a National Science Foundation Graduate Research Fellowship. synchronization and reproducibility. During the initial running of 2009 and 2010, changes in the luminous region parameters were monitored in real-time by the ATLAS High-Level Trigger system (HLT) and provided the opportunity to relay information about the LHC beams to both the ATLAS and LHC control room operators, providing important feedback on the stability of the position and size of the interaction region and luminosity.

Using charged-particle tracks emerging from $p p$ collisions and measured by the ATLAS Inner Detector we reconstruct vertices on an event-by-event basis within the HLT. The threedimensional distribution of these vertices reflects that of the luminosity and can be parametrized by a three-dimensional Gaussian whose paramters are sometimes referred to as the luminous ellipsoid, or also as the "beam spot". The coordinates of its luminous centroid determine, in the ATLAS coordinate system, the position of the average collision point; the orientation of the luminous ellipsoid in the horizontal $(x-z)$ and vertical $(y-z)$ planes is determined by the angles and relative transverse sizes of the two beams at the IP; and the transverse and longitudinal dimensions of the luminous region, quantified in terms of the luminous sizes, are related to the corresponding IP sizes of the two beams.

These observables are continuously reconstructed and monitored online in the HLT, and communicated to the control room displays every few minutes. In addition, the instantaneous rate of reconstructed vertices can be used online as a luminosity monitor. Following these online measurements a system for applying real-time configuration changes on the High-Level Trigger farm is utilized for distributing the updates in a way that does not disrupt data taking or incur deadtime.

\section{ATLAS DETECTOR AND TRIGGER SYSTEM}

The innermost part of the ATLAS detector [3] used for this analysis is a silicon tracker, comprised of a pixel detector at the center and a silicon strip detector (SCT) surrounding it. The pixel detector consists of three barrel layers, the innermost one at $5 \mathrm{~cm}$ from the interaction region, and three disks at each endcap for a total of over 80 million pixel channels, mostly $50 \mu \mathrm{m} \times 400 \mu \mathrm{m}$ in size. The SCT adds four double layers of silicon micro-strips in the barrel, and nine disks on each side that make up the endcap, and contains 8 million channels. A transition-radiation tracker (TRT) surrounds the silicon detectors and is comprised of up to 76 layers of longitudinal straw tubes in the barrel and 160 radial straw planes in each endcap. Hits in the TRT are used to improve the momentum resolution of tracks found using the Pixel and SCT. These three devices are immersed in a $2 \mathrm{~T}$ solenoidal magnetic 
field and are optimized to provide excellent tracking efficiency and resolution over a pseudo-rapidity range of $|\eta|<2.5$.

The ATLAS trigger system [4] performs the online event selection in three stages, referred to as Level 1 (L1), Level 2 (L2) and Event Filter (EF). L2 and EF are collectively referred to as the High-Level Trigger (HLT) and are based on software algorithms running on large farms of commercial processors. L2 is the first stage of the ATLAS data acquisition system that has access to data from the inner detectors and is capable of doing partial reconstruction of events at combined rates of up to $75 \mathrm{kHz}$.

The particular place of the L2 trigger in the data acquisition chain and its capability to reconstruct tracks and vertices online give it the unique capability of producing results in realtime, i.e. with minimal latency, as well as the advantage of the high statistics of the early trigger stage, including events that are subsequently rejected from the data logging and thus not available for offline analysis. Furthermore, by restricting the event data written to disk - a procedure referred to as partial event building - a large fraction of the events used for online vertex finding can be made available offline for dedicated calibration and bias studies. This is primarily achieved by saving only the inner detector space point information, thereby reducing the data volume by a factor of approximately 15 . The monitoring infrastructure of the HLT supports the real-time accumulation of histograms and their aggregation across the farm, so that parameters can be extracted from cumulative distributions that contain events from all processor nodes. Beam parameters determined from those live histograms are available in the control room and are also available for feed back into the HLT itself for use by trigger algorithms that depend on the precise knowledge of the luminous region (such as $b$-jet tagging).

\section{VERTEX RECONSTRUCTION}

We use the distributions of event vertices collected over short time intervals to measure the position and shape of the luminous region. These vertices are formed from tracks reconstructed at L2 using specialized, fast algorithms, optimized for the average processing time constraint of only a few tens of milliseconds. The pattern recognition is based on the silicon detectors (the Pixel and SCT), and tracks found there are subsequently extended to the TRT in order to improve the momentum measurement ("inside-out" strategy). The full details of the L2 tracking algorithms and their performance in the first $\sqrt{s}=900 \mathrm{GeV}$ data are described in these proceedings [5].

The online beam spot algorithm employs a fast vertex fitter [6] able to efficiently fit the L2 tracks emerging from the interaction point to a common vertex within a fraction of the time budget. Tracks are first grouped into clusters with similar impact parameter $\left(z_{0}\right)$ along the nominal beam-line at the center of ATLAS. A track- $p_{\mathrm{T}}$ ordered list is formed and a cluster of tracks is initiated with the highest- $p_{\mathrm{T}}$ track above $0.7 \mathrm{GeV}$ in the event. The input to the vertex fitter is assembled from this "seed" track and the neighboring tracks with $p_{\mathrm{T}}>0.5 \mathrm{GeV}$ and within $\Delta z_{0}<1 \mathrm{~cm}$ of the seed

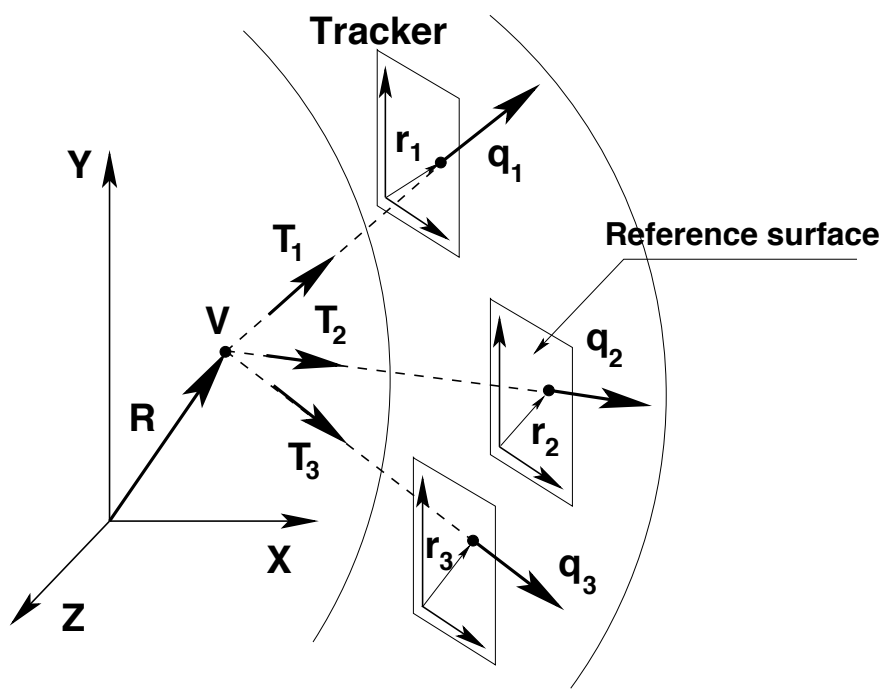

Fig. 1. Fast vertex reconstruction using track measurements.

track. The average $z$-centroid of the track cluster is then used as a starting point for the vertex fitter in the longitudinal direction. The vertex fitter itself employs a decorrelating matrix that allows for using the track parameters as measured at a given reference surface, as shown in Fig. 1, instead of inferring those parameters at the position of the vertex (a time consuming procedure). This choice significantly reduces the computational burden of the fitting procedure and results in a robust algorithm that fully satisfies the time constraints imposed at L2. All tracks are required to have at least four silicon space points, with at least one in the Pixel detector and three in the SCT. A very loose selection on the transverse impact parameter with respect to the nominal beam-line of $\left|d_{0}\right|<1 \mathrm{~cm}$ is applied. To maximize the available statistics in the early, low-intensity running, we reconstruct vertices with two or more tracks of $p_{\mathrm{T}}>1 \mathrm{GeV}$ covering a rapidity range of $|\eta|<2.5$. This selection has been tightened for the higher center-of-mass energy during 2010.

\section{Luminous REgion MEASUREMENT}

Using the event-by-event vertex distribution computed in real-time by the HLT and accumulated in intervals of typically two minutes, we measure the position, size and tilt angles of the luminous region within the ATLAS coordinate system. A view of the transverse distribution of vertices reconstructed by the HLT in real-time is shown in Fig. 2 for both $\sqrt{s}=$ $900 \mathrm{GeV}$ and $\sqrt{s}=7 \mathrm{TeV}$.

Fig. 3 shows the transverse and longitudinal positions of reconstructed vertices at both $\sqrt{s}=900 \mathrm{GeV}$ and $\sqrt{s}=$ $7 \mathrm{TeV}$. In addition to a difference in the transverse luminous centroid (the mean $x$ or $y$ position), a very clear change in the width of the distribution is apparent between the two center-ofmass energies, even before correcting for the intrinsic vertex resolution. At the higher center-of-mass energy $(\sqrt{s}=7 \mathrm{TeV})$, the reduction in transverse luminous width associated with the energy increase is striking; it is dominated by the change in geometrical emittance of the LHC beam [7], which scales as the inverse of the beam energy. 




(a) Primary vertex $x(900 \mathrm{GeV})$.

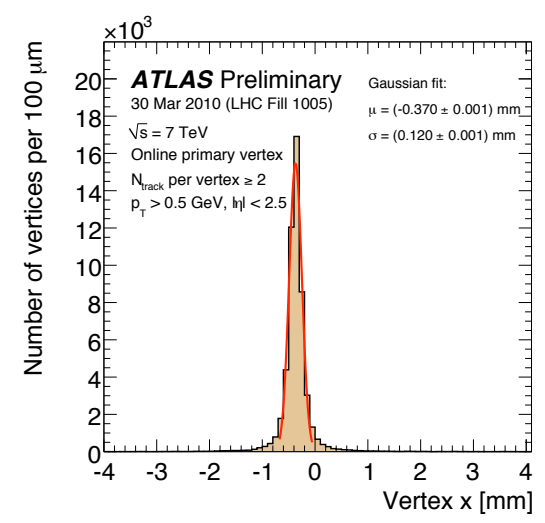

(d) Primary vertex $x(7 \mathrm{TeV})$.

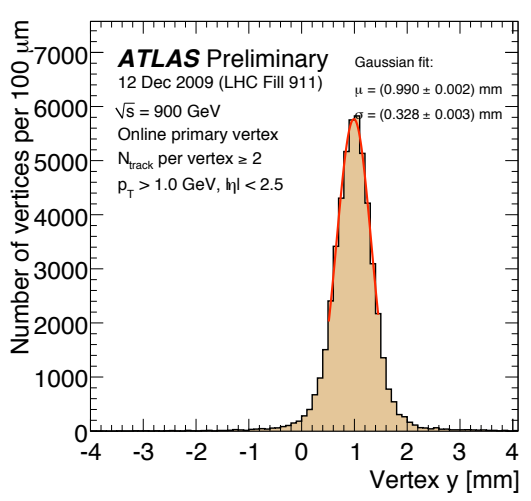

(b) Primary vertex $y(900 \mathrm{GeV})$.

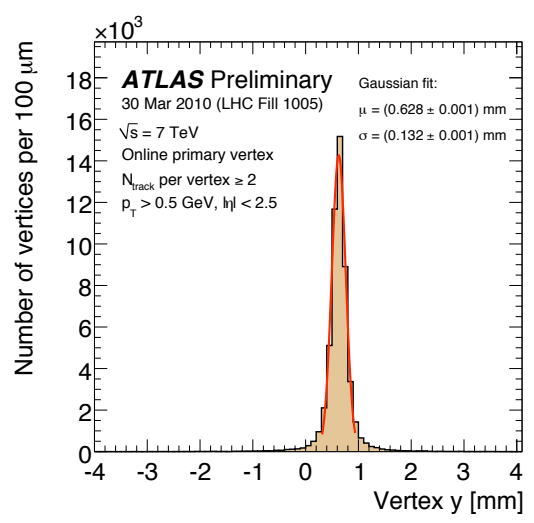

(e) Primary vertex $y(7 \mathrm{TeV})$.

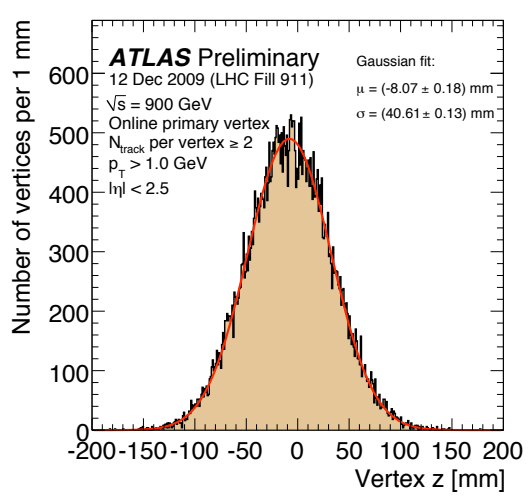

(c) Primary vertex $z(900 \mathrm{GeV})$.



(f) Primary vertex $z(7 \mathrm{TeV})$.

Fig. 3. Primary vertex distributions at $\sqrt{s}=900 \mathrm{GeV}$ and $\sqrt{s}=7 \mathrm{TeV}$ as produced by the ATLAS HLT, before correcting for vertexing resolution. Gaussian fits are used to extract the mean position and observed width; the transverse fit range is limited to \pm 1 RMS to reduce the sensitivity to resolution tails.

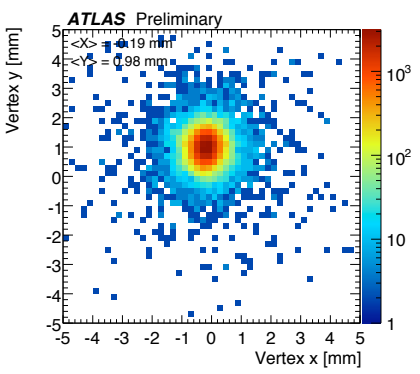

(a) $\sqrt{s}=900 \mathrm{GeV}$.

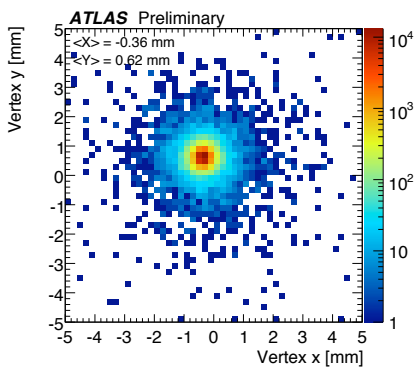

(b) $\sqrt{s}=7 \mathrm{TeV}$ ).

Fig. 2. Transverse view of the LHC luminous region as reconstructed online using event-by-event vertices in the HLT at both (a) $900 \mathrm{GeV}$ and (b) $7 \mathrm{TeV}$.

The time-evolution of the luminous centroid position is illustrated in Fig. 4 for part of the $900 \mathrm{GeV}$ running period. With the exception of a couple of step changes that can be associated with beam-orbit readjustments, the transverse luminous centroid remained within at most a $\pm 25 \mu \mathrm{m}$ envelope over a two-week period, implying a stability of the orbit at the level of a few percent of the IP beam size at $450 \mathrm{GeV}$ $(\sim 280 \mu \mathrm{m})$.

The vertex-based determination of the luminous centroid is also compared to the longitudinal position of the collision point extracted from the arrival times of the colliding bunches,

as measured via time-of-flight measurements by electrostatic beam-pickup detectors (BPTX) [8] in Fig. 4. These pick-ups are installed as part of the LHC beam instrumentation on either side of the ATLAS detector along the beam-line and used by ATLAS for timing and beam-monitoring purposes. After accounting for cable-length delays in the signal propagation, an approximate $100 \mathrm{ps}$ timing resolution is achieved and the measured longitudinal collision point from the HLT vertex fitting is found to be in good agreement with that expected from the BPTX, demonstrating the real-time merit of this approach.

In very early running, the longitudinal crossing point was observed several centimeters off the nominal IP-position (not shown); it was promptly moved within a few $\mathrm{mm}$ of its nominal position by adjusting the relative RF phase between the two LHC rings, and thereafter remained stable within a few $\mathrm{mm}$. The longitudinal position of the collision point was further adjusted in $7 \mathrm{TeV}$ running to be nearly perfectly centered, as shown in the longitudinal vertex distribution of Fig. 3(f) which was used online to verify the RF adjustments in-situ.

The orientation of the luminous ellipsoid in the horizontal and vertical planes can be measured in the ATLAS coordinate system by slicing the transverse vertex distribution in bins along the $z$ axis, and performing Gaussian fits to the horizontal 

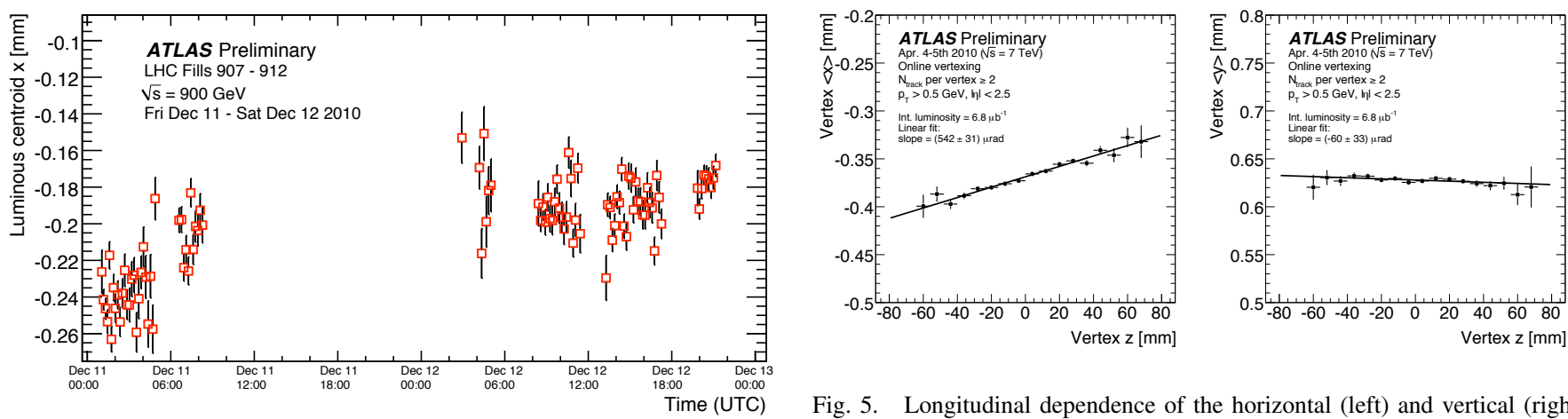

Fig. 5. Longitudinal dependence of the horizontal (left) and vertical (right) position of the luminous centroid, measured online at $7 \mathrm{TeV}$. The errors are

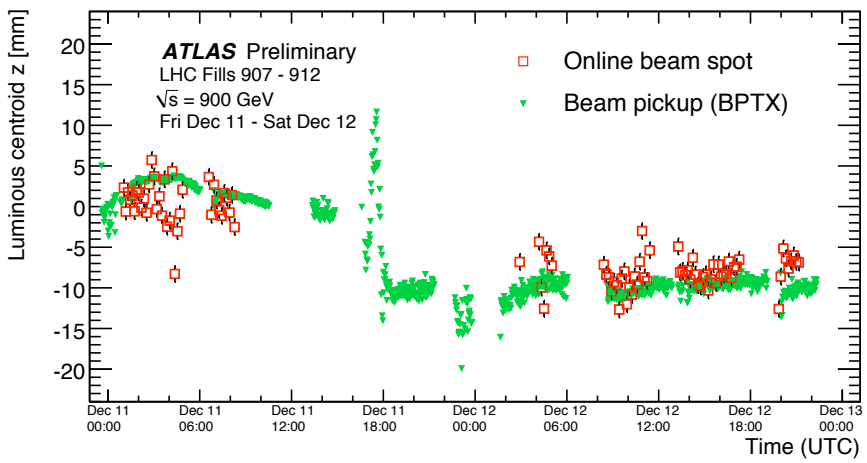
statistical only.

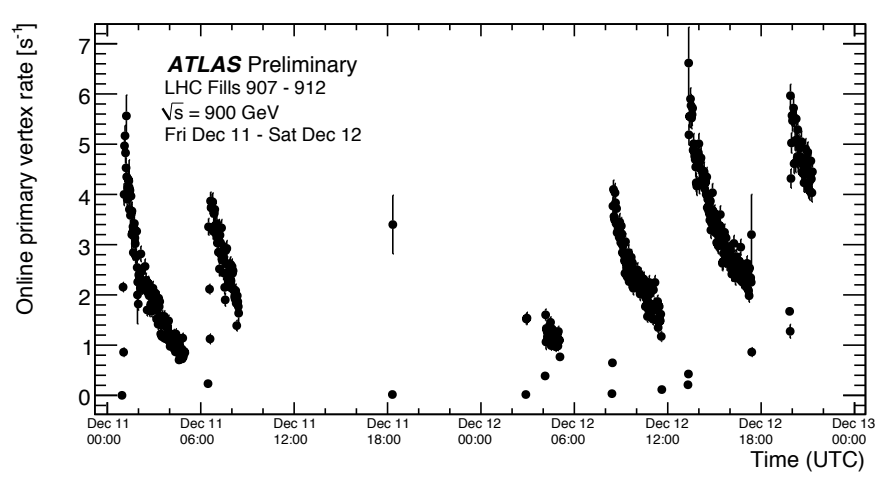

Fig. 6. Time history of the primary-vertex rate recorded by the ATLAS High-Level Trigger at $\sqrt{s}=900 \mathrm{GeV}$.

fill. The vertex-counting rate is also compared in detail to the luminosity measured offline using the ATLAS Liquid Argon calorimeter (LAr) for a single fill in Fig. 7. The LAr measurement is based on events satisfying one of the minimumbias trigger selections and on minimum energy depositions in electromagnetic calorimeter cells covering the pseudo-rapidity range $2.5<|\eta|<4.9$. Requiring a time difference of $|\Delta t|<$ $5 \mathrm{~ns}$ between cells on the two sides effectively eliminates beam-halo triggers and provides a very-high purity sample of collision events. The systematic uncertainty on the absolute luminosity from this method is about $20 \%$, dominated by the difference in physics modeling by various event generators.

Since the reconstruction of event vertices is effectively restricted by the inner detector acceptance to the range of $|\eta|<2.5$ - complementary to the acceptance of the LAr selection detailed above - the two samples are built from a rather different mix of non-diffractive, single-diffractive and doublediffractive events. The time-evolutions of the two luminosity measurements follow each other precisely, suggesting that the background is very low in both samples (or that in both cases it represents a constant fraction of the collision rate).

Beam-separation scans, originally proposed by S. van der Besides monitoring the luminous region parameters during routine physics running, the reconstructed vertices can be used online as an additional luminosity monitor, which can be calibrated together with the other ATLAS luminosity monitors using beam-separation scans.

The time-history of the online vertex-counting rate, over several fills at $\sqrt{s}=900 \mathrm{GeV}$, is presented in Fig. 6 and clearly exhibits the typical luminosity decay within a given Meer [9] and routinely used at most high-energy particle colliders, provide a simple method for calibrating the absolute luminosity by measuring simultaneously the collision rate and the fundamental accelerator parameters that determine the luminosity.

A preliminary luminosity scan was performed at the ATLAS 


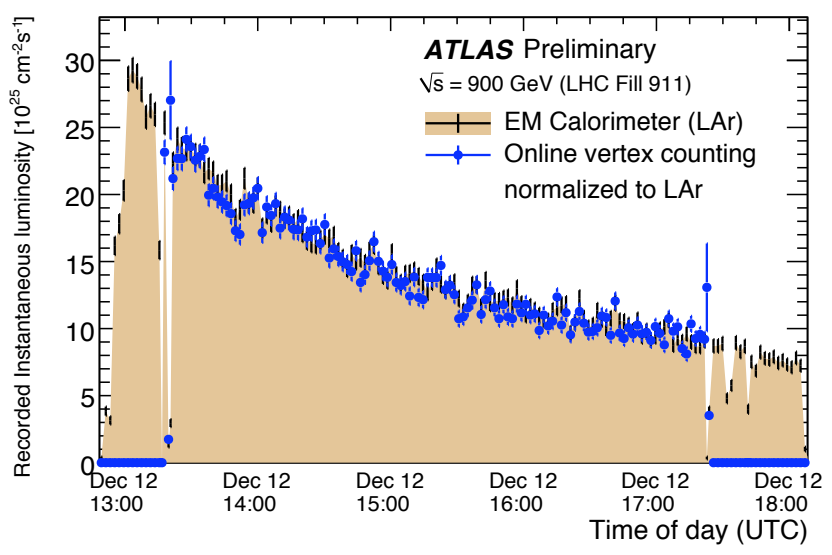

Fig. 7. Luminosity measurement by vertex-counting, compared to the LiquidArgon (LAr) calorimeter measurement for LHC fill number 911 (Dec 12, 2009) at $\sqrt{s}=900 \mathrm{GeV}$. The vertex-counting rate is normalized to the LAr luminosity using only the time bin with the largest number of reconstructed vertices, i.e. with the highest luminosity. The online beam spot algorithm in the HLT is only active in the period of stable beams when the Pixel and SCT detectors are both ramped up.

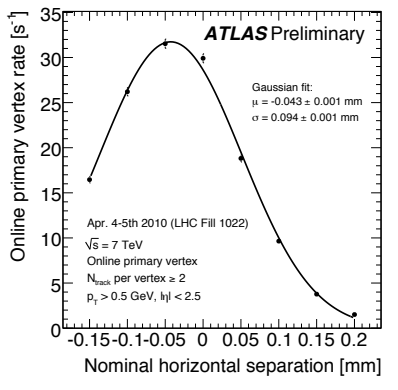

(a) Horizontal luminosity scan

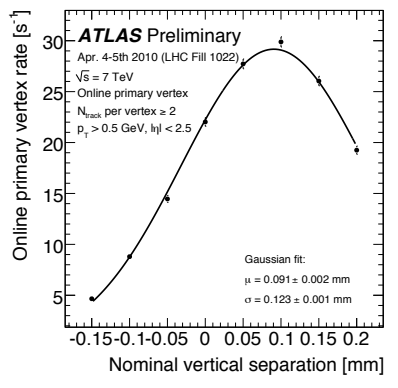

(b) Vertical luminosity scan
Fig. 8. Luminosity scan data for the (a) horizontal and (b) vertical planes using online primary vertex-counting.

IP using the $3.5 \mathrm{TeV}$ beams in April 2010. We monitored the centroid positions and the interaction rate via vertex-counting as a function of the nominal horizontal and vertical beam separations programmed into the accelerator control system. The IP positions of each beam, and their separation (up to an arbitrary relative offset) were also recorded using the beamposition monitors (BPMs) located $\pm 21.5 \mathrm{~m}$ from the ATLAS IP, on the IP-side of the final-triplet quadrupoles. A total of 9 separation steps were performed in each of the horizontal and vertical planes, of which 7 or 8 points were used for the luminosity calibrations.

The online measurements performed during this scan, which provided fast feedback on both luminous-centroid positions and vertex rates in two-minute intervals, are used to measure the convolution integral of the two beams (Fig. 8) in both the horizontal and vertical dimensions, thus demonstrating the ability to measure these quantities in real-time using the HLT.

\section{ReAL-Time CONFIGURATiOn UPdATES}

One of the primary uses of the online luminous region measurement is for L2 $b$-jet trigger. These $b$-tagging trigger algorithms are designed to select events with long-lived $b$ quarks which typically hadronize and decay a significant distance from the primary proton-proton interaction. In order to measure the significance of this decay length, a precise knowledge of the LHC interaction region is crucial. Any changes or drifts in the average interaction point (as already discussed in Section IV) must be fed back to the $b$-tag algorithms to maintain a high efficiency for selecting real $b$ decays and good rejection against background events.

A dedicated system exists to allow the real-time configuration changes of the High-Level Trigger farm that deliver this information to several hundred processing nodes in a way that does not disrupt data taking or incur deadtime and ensures a consistent and reproducible configuration across the farm. A so-called "proxy-tree" allows for caching of information at the level of each node, as well as at the level of groups of nodes (rack-level, consisting of 30 nodes), and top level set of rack proxies. This allows to reduce the load on the database when large updates are made and vastly improves the speed for distributing these updates to each node. This system depends on the usage of so-called "luminosity blocks" (LB) which are the intervals on the order of two minutes during the run which define a period of approximately constant luminosity and detector conditions, and which are used as the monitoring interval of the luminous region measurements. This infrastructure is described in detail in [10] in these proceedings, and the procedure relevant to the redistribution of the luminous region parameters (the "beam spot") to the the High-Level Trigger can be summarized as follows:

1) Vertex distributions are accumulated in the form of histograms from the HLT farm and the luminous region parameters are extracted from these histograms via fits.

2) A comparison is made with respect to the previously determined luminous region parameters (available in the distributed online ATLAS conditions database).

3) If a significant change is detected, new parameters are written to the conditions database with an interval of validity starting from LB $N+2, N$ being the current luminosity block, and valid to infinity ("open-ended").

4) The LB at which the detector configuration is intended to change $(N+2)$ is then written into the data fragment delivered to each node of the HLT farm for each event and which contains the current LB number as well as the LB number for which an update is pending.

5) The HLT applications check this data fragment, and if a configuration change is detected, the new configuration is read from the conditions database.

It is useful to add that only the node receiving the first event of LB $N+2$ is required to initiate the database access, thereby filling the proxy caches at both the node and the proxy levels above that node. The time for this access has been measured approximately a few milliseconds, comparable to or less than the average processing time of one node. All subsequent nodes then receive the information from the proxy caches, which is essentially instantaneous. By delivering the information of which luminosity block contains the updated conditions information directly in the data fragment we ensure 
the second requirement that this approach be synchronous across the farm. Finally, the fact that the parameters are first written to the conditions database (which is also subsequently available offline) and then read from the nodes makes then entire procedure strictly reproducible

In this way, the real-time updates to the HLT configuration for the LHC luminous region are guaranteed to not incur deadtime, be synchronous across the farm, and reproducible.

\section{CONCLUSIONS}

We have used the ATLAS Inner Detector and High-Level Trigger infrastructure to measure luminous-region parameters of the LHC and their time evolution in real-time. Primary vertex distributions were reconstructed online to extract position, sizes and tilt angles, as well as instantaneous event rates. These measurements could be correlated with LHC beam instrumentation data such as from electrostatic beam-pickup monitors. By measuring the rate of reconstructed vertices during beam separation scans, initial estimates of the convolution integral of the two beams was extracted. Updates of the lumious-region conditions, used by the HLT processors for e.g. $b$-tagging, are being delivered to the farm through a mechanism that does not disrupt data taking or incur deadtime and achieves full synchronicity and reprodicibility of the configuration changes. This system has been tested and is currently being fully deployed in the online system.

\section{ACKNOWLEDGMENT}

We would like to thank the LHC coordination team and the LHC beam commissioning working group for providing access to the machine instrumentation data and for planning and carrying out the luminosity scans.

\section{REFERENCES}

[1] G. Aad et al., "The ATLAS Experiment at the CERN Large Hadron Collider," JINST, vol. 3, p. S08003, 2008.

[2] L. Evans and P. Bryant, "LHC Machine," JINST, vol. 3, p. S08001, 2008.

[3] G. Aad et al., "The ATLAS Inner Detector commissioning and calibration," accepted by EPJC, 2010.

[4] G. Aad et al., "Expected Performance of the ATLAS Experiment Detector, Trigger and Physics," no. CERN-OPEN-2008-020, 2009.

[5] I. A. Christidi, "Performance of the ATLAS Inner Detector Trigger algorithms in p-p collisions at $\sqrt{s}=900 \mathrm{GeV}$," in these proceedings, 2010.

[6] D. Emeliyanov, "A fast vertex fitter for ATLAS level 2 trigger," PoS, vol. ACAT, p. 058, 2007.

[7] W. Kozanecki et al., "Interaction-Point Phase-Space Characterization using Single-Beam and Luminous-Region Measurements at PEP-II," Nucl. Instrum. Meth., vol. A607, pp. 293-321, 2009.

[8] C. Ohm and T. Pauly, "The ATLAS beam pick-up based timing system," NIM A, vol. In Press, 2010

[9] S. van der Meer, "Calibration of the effective beam height in the ISR," CERN, Geneva, Tech. Rep. CERN-ISR-PO-68-31, 1968.

[10] F. Winklmeier, "Real-time conguration changes of the ATLAS High Level Trigger," in these proceedings, 2010. 